\title{
2 I 0 I Role of hemodynamic forces on plaque development: non-invasive assessment with MRI methods
}

\author{
Jie Zheng*1, Dana R Abendschein1, Ruth J Okamoto1, Deshan Yang1, \\ Kyle S McCommis ${ }^{1}$, Bernd Misselwitz ${ }^{2}$, Robert J Gropler ${ }^{1}$ and Dalin Tang ${ }^{3}$
}

\author{
Address: ${ }^{1}$ Washington University, St. Louis, MO, USA, ${ }^{2}$ Bayer Schering Pharma AG, Berlin, Germany and ${ }^{3}$ Worcester Polytechnic Institute, \\ Worcester, MA, USA \\ * Corresponding author
}

from I I th Annual SCMR Scientific Sessions

Los Angeles, CA, USA. I-3 February 2008

Published: 22 October 2008

Journal of Cardiovascular Magnetic Resonance 2008, I0(Suppl I):A370 doi:I0.I 186/I532-429X-10-SI-A370

This abstract is available from: http://jcmr-online.com/content/I0/SI/A370

(c) 2008 Zheng et al; licensee BioMed Central Ltd.

\section{Purpose}

Fluid shear stress was thought to lead to atherosclerotic plaque progression, but such measurement procedures are often invasive. The goal of the present study is to address the biomechanical pathways of atherosclerosis progression and regression with our non-invasive MRI methods and a 3D fluid-structure interaction (FSI) model. The study was performed in a large animal atherosclerotic model.

\section{Methods}

Five pigs were fed cholesterol-enriched diets (HC) and then underwent balloon overstretch injury to the left carotid artery to simulate angioplasty or stent restenosis. A plaque-targeting contrast agent, Gadofluorine (Bayer Schering Pharma AG, Germany) enhanced MRI scans were performed at various time points after the balloon injury while the pigs were maintained on HC. The atherosclerotic-like carotid lesions in four of the pigs were then re-injured by balloon angioplasty. MRI scans were repeated to monitor the progression and regression (by feeding normal chow diet to the pigs) of the plaques. The pigs were euthanized after 5 to 9 months and the left and right (normal) carotid arteries were dissected for histopathology and mechanical testing to obtain subject-specific material properties.

In each MRI study, Gadofluorine was injected intravenously at a dose of $0.05-0.075 \mathrm{mmol} / \mathrm{kg}$ one hour before each MRI scan. The MRI (at 3-T, Siemens Trio MRI System) protocol consisted of 20-slice T1w and T2w imaging using dark-blood turbo-spin-echo (DB-TSE) sequence, as well as a 20-slice 3D gradient-echo (GRE) imaging. The slice thickness was $2 \mathrm{~mm}$ and the true image resolution was $0.33 \mathrm{~mm} \times 0.33 \mathrm{~mm}$. The left carotid vessel wall and plaque images were segmented based on contrast enhancement patterns. Using the material parameters obtained from mechanical testing, the 3D FSI computational model that was validated in an in vitro study was applied to data from two pigs to calculate various structure stress and strain distribution, including maximal fluid shear stress, within the plaques at different time points.

\section{Results}

(1) Gadofluorine enhancement of plaque, which has been associated with extracellular matrix, was increased by $60-100 \%$ compared with non-enhanced plaque areas. The use of three-contrast images helped to define the plaque components: 3D GRE images clearly delineated the out-boundary of the vessel wall and DB-TSE images unambiguously defined the inner-boundary and contrast enhanced plaque areas (Figure 1). Progression and regression of these components could be readily demonstrated.

(2) With subject-specific plaque material parameters and greatly improved plaque image quality, structure stress/ strain within the left carotid plaques was calculated. Flow 

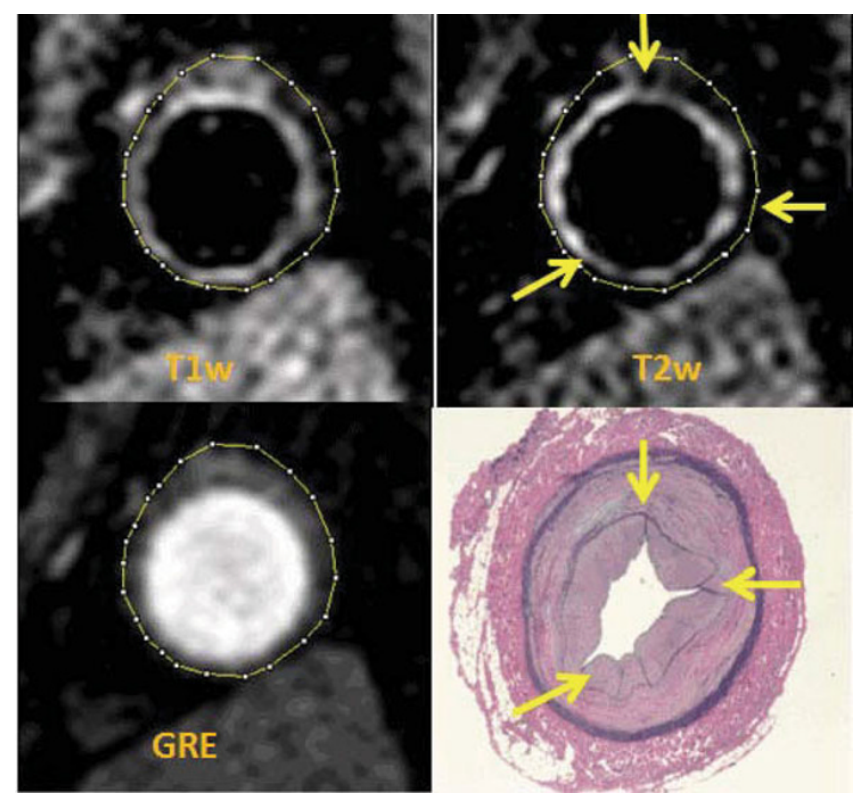

shear stress and structure stress/stain were shown to correlate interestingly with the change in the plaque dimension during plaque progression and regression (Figure 2).

\section{Conclusion}

Gadofluorine enhanced MRI and 3D computational models may allow non-invasive dynamic assessment of biomechanical behaviors of atherosclerotic plaques in vivo. Structure stress and strain may play an important stimulation role for plaque regression. This more comprehensive information would provide useful insight to the plaque development and help select appropriate interventions to avoid subsequent cardiac events.

\section{Figure I}

Different MRI contrasts to identify proliferative intima (hyperintensity, T2w) media (hypointensity, T2w) and lumen (hyperintensity GRE). The pathology shows corresponding neotimal formulation.

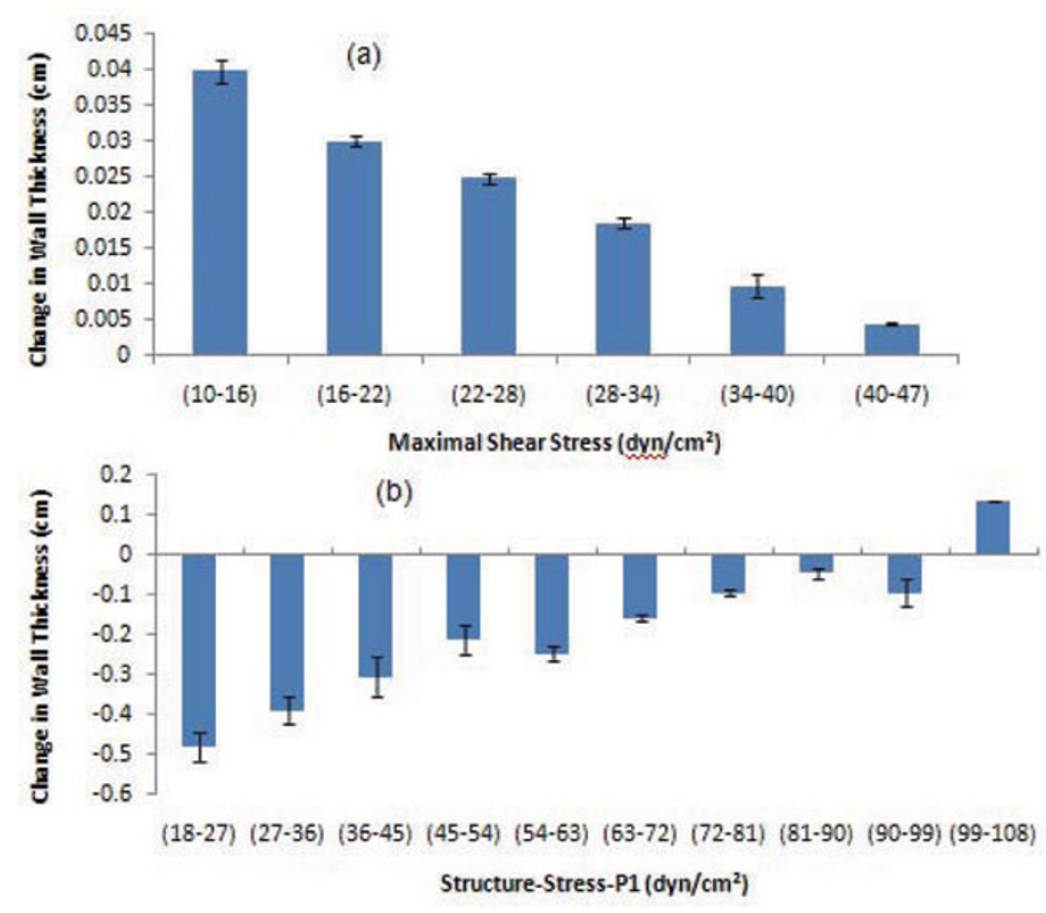

\section{Figure 2}

(a) Lower MSS leads to faster plaque growth in pig I during a progression; (b) Lower structure stress-PI leads to faster plaque reduction in pig 2 during regression. 